\title{
Wavelength-coded volume holographic imaging endoscope for multidepth imaging
}

Isela D. Howlett

Wanglei Han

Photini Rice

Jennifer K. Barton

Raymond K. Kostuk 


\section{Wavelength-coded volume holographic imaging endoscope for multidepth imaging}

\author{
Isela D. Howlett, ${ }^{a, b, *}$ Wanglei Han, ${ }^{a, b}$ Photini Rice, ${ }^{c}$ \\ Jennifer K. Barton, ${ }^{a, b, c}$ and Raymond K. Kostuk ${ }^{a, b}$ \\ aniversity of Arizona, College of Optical Sciences, Tucson, Arizona, \\ United States \\ bUniversity of Arizona, Department of Electrical and Computer \\ Engineering, Tucson, Arizona, United States \\ 'University of Arizona, Department of Biomedical Engineering, \\ Tucson, Arizona, United States
}

\begin{abstract}
A wavelength-coded volume holographic imaging (WC-VHI) endoscope system capable of simultaneous multifocal imaging is presented. The system images light from two depths separated by $100 \mu \mathrm{m}$ in a tissue sample by using axial chromatic dispersion of a gradient index probe in combination with two light-emitting diode sources and a multiplexed volume hologram to separate the images. This system is different from previous VHI systems in that it uses planar multiplexed gratings and does not require curved holographic gratings. This results in improved lateral imaging resolution from 228.1 to $322.5 \mathrm{lp} / \mathrm{mm}$. This letter describes the design and fabrication of the WC-VHI endoscope and experimental images of hard and soft resolution targets and biological tissue samples to illustrate the performance properties. @ 2017 Society of Photo-Optical Instrumentation Engineers (SPIE) [DOI: 10.1117/1.JBO.22.10 .100501]
\end{abstract}

Keywords: optics; endoscopy; holography; chromatic dispersion; volume holographic imaging.

Paper 170440LR received Jul. 5, 2017; accepted for publication Sep. 13, 2017; published online Oct. 2, 2017.

Optical instruments capable of microscopic imaging of tissue samples during surgical procedures can provide important information for the detection of cancer and other life-threatening diseases. Confocal microscopy and optical coherence tomography methods are capable of providing optical sectioning during in vivo procedures. ${ }^{1,2}$ However, drawbacks to these systems include long image capture times and mechanical scanning requirements that increase system complexity, cost, and weight. Introduction of volume holographic imaging (VHI) techniques, a method analogous to slit scan confocal microscopy, has shown the capability of simultaneous multidepth imaging. ${ }^{3-6}$ VHI imaging removes the need for mechanical scanning through the use of multiplexed volume holographic elements. ${ }^{7-15}$

Of particular interest in the development of VHI systems is the use of wavelength-coded methods to address the issue of

*Address all correspondence to: Isela D. Howlett, E-mail: idh @email.arizona .edu contrast reduction caused by the hologram channel crosstalk and hologram Bragg degeneracy properties. ${ }^{16,17}$ In previous wavelength-coded VHI (WC-VHI) designs, a separate wavelength and wavefront curvature is encoded in each hologram. The wavefront curvature recorded in the hologram selects light from a specific depth within the tissue as in the standard reflectance mode VHI system. The different encoded wavelengths do two things. First, the separation between wavelengths is large enough so that there is essentially no crosstalk between image channels. The second advantage is that the intensity of each source can be independently adjusted to equalize the brightness of each image. This improves image quality; however, Bragg degeneracy still exists, which limits contrast when light emitting diodes (LEDs) are used as the optical sources. In addition, since the holograms have curved fringes and are recorded at different wavelengths than the reconstruction wavelength, chromatic aberration results along the dispersive axis and reduces image resolution.

In this work, a WC-VHI endoscope system that uses wavelength coding in combination with the axial chromatic dispersion of a gradient index (GRIN) probe to eliminate the need for curved fringe holograms is described. This is accomplished by using the axial chromatic dispersion to focus light from the two wavelengths at separate depths. Since the two wavelengths are sufficiently separated, a planar grating can be used to diffract each wavelength band. Since planar gratings can be Bragg matched when the recording and reconstruction wavelengths are different, the image resolution does not degrade. Chromatic dispersion has been used for depth sectioning in other systems such as confocal microendoscope designs. $^{18-20}$

Figure 1 shows the schematic of the endoscope probe indicating the imaging planes within the sample and the overlap of the images at the output for viewing by the microscope objective. In the WC-VHI endoscope, three 2.7-mm diameter GRIN lenses and two 3.0-mm diameter windows are used to form the probe, which is essentially a unit magnification relay. The LED sources used for this experiment have peak wavelengths of 660 and $730 \mathrm{~nm}$ with a full width half maximum of $20 \mathrm{~nm}$. The resulting total axial chromatic separation between the two focal planes for the two wavelengths is $100 \mu \mathrm{m}$. The overlap of the surface and depth at the output of the endoscope probe is due to the axial chromatic dispersion of the endoscope optics.

Figure 2 shows the experimental set up of the WC-VHI system using planar gratings to separate the surface (blue) and depth (red) images. The holographic element is placed after a relay (not shown) such that the hologram pupil is matched to the objective lens pupil. The WC-VHI system is designed such that the surface channel information is encoded in a hologram, which reconstructs with the 660-nm wavelength source, and the depth channel is encoded with the 730-nm source. Each hologram in the multiplexed element diffracts light returning from the sample at a different wavelength in a different direction so that the two images are separated on the camera aperture. The axial point spread function was measured using the 660- and 730-nm LED illumination sources and found to be $\sim 0.50 \mathrm{~mm}$ for both sources. This is consistent with degenerate VHI as described by Castro et al. ${ }^{3}$

A Coherent Innova 300 Argon laser, $514.5 \mathrm{~nm}$, was used to record the volume holograms. The volume holograms were

1083-3668/2017/\$25.00 @ 2017 SPIE 


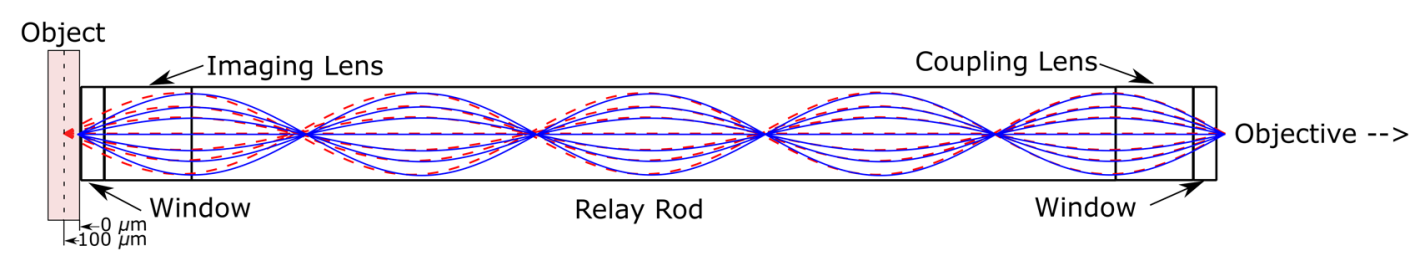

Fig. 1 The schematic of the endoscope assembly depicts the focal plane positions in the object for the surface and $100 \mu \mathrm{m}$ within the sample. Each focus region corresponds to a specific wavelength based on the chromatic effects of the endoscope probe. In this configuration, the blue (solid) line corresponds to the 660-nm LED source and the red (dashed) line corresponds to the 730-nm LED source.

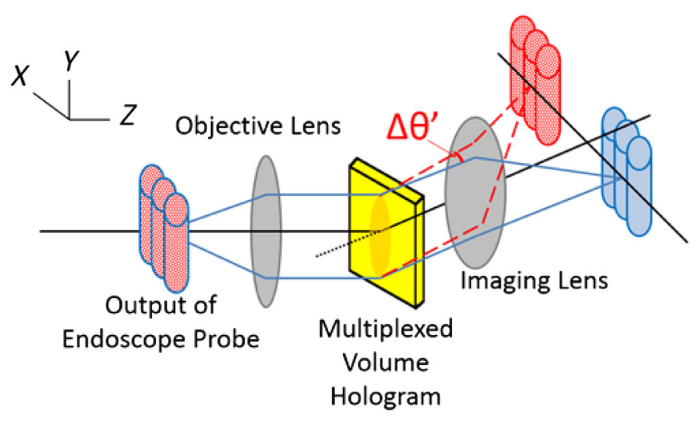

Fig. 2 A schematic of the WC-VHI system showing the spectral separation of the surface and depth images by the multiplexed holographic element. Surface and depth information overlaps prior to the hologram. One of the multiplexed holograms diffracts the 660$\mathrm{nm}$ light from the surface position on the sample, and the second hologram diffracts the 730-nm light from the depth position.

recorded in a 1.8-mm thick $(d)$ sample of phenanthraquinonedoped poly methacrylate that has an index of refraction of $n=1.49$. The holograms were designed as thick volume holograms to provide high diffraction efficiency in a single diffraction order with high angular and wavelength selectivity. ${ }^{21,22}$ The grating periods for the surface and depth holograms are $\Lambda_{\text {surface }}=460.8 \mathrm{~nm}$ and $\Lambda_{\text {depth }}=525.2 \mathrm{~nm}$ and have $Q$ parameters, $Q=\frac{2 \pi \lambda d}{n \Lambda^{2}}$, that are significantly greater than 10 and well within the volume hologram regime. The holograms are phase transmission gratings with a diffraction efficiency for volume phase transmission holograms given by $\eta=\frac{\sin ^{2}\left(\nu^{2}+\xi^{2}\right)^{\frac{1}{2}}}{\left(1+\frac{\xi^{2}}{\nu^{2}}\right)}$ (with $\quad \nu=\frac{\pi n_{1} d}{\lambda c_{r} c_{s}} ; \quad \xi=\frac{\vartheta d}{2 c_{s}}$ ), where $\quad c_{r}=\cos \left(\theta_{r}\right) \quad$ and $c_{s}=\cos \left(\theta_{s}\right)$, where $\theta_{r}$ and $\theta_{s}$ are the reconstruction and diffracted beam angles ${ }^{21}$ respectively, $d$ is the hologram thickness, and $n_{1}$ is the refractive index modulation. The diffraction

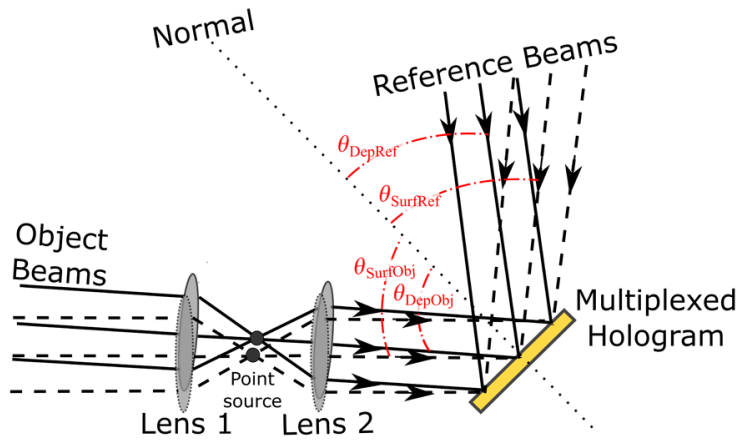

Fig. 3 Hologram recording geometry for the surface (dashed) and depth (solid) multiplexed gratings. efficiency of the surface hologram was $27 \%$ at $660 \mathrm{~nm}$ and $39 \%$ for the depth hologram at $730 \mathrm{~nm}$. The construction angles for the object and reference beams with 514.5-nm light are determined from the $K$-vector or Bragg matching relation: $\vec{K}=\vec{k}_{\text {ref }}-\vec{k}_{\text {obj }}$, where $\vec{k}_{\text {ref }}$ and $\vec{k}_{\text {obj }}$ are the reference and object propagation vectors, respectively, and $\vec{K}$ is the grating vector, which has a magnitude of $\frac{2 \pi}{\Lambda}$. The object beam angles are $33 \mathrm{deg}$ for the surface channel and $30 \mathrm{deg}$ for the depth channel, in air relative to the normal to the hologram surface. The reference beam angles for the surface and depth channels are $34.8 \mathrm{deg}$ and $28.6 \mathrm{deg}$, respectively. These angles were derived from reconstruction of the surface channel with 660-nm light and the depth channel at $730 \mathrm{~nm}$. A general schematic of the recording setup is depicted in Fig. 3.

A USAF 1951 bar target is used to assess the overall system dependence on the light sources, the hologram performance, and the image resolution. To verify that crosstalk between imaging channels is negligible, each source is turned on separately and the signal is viewed on the camera. The resulting effect using the 660-nm source is shown in Fig. 4(a) and using the 730-nm source is shown in Fig. 4(b). It is seen that when the 660 -nm source is turned on, little to no signal reaches the camera from the 730-nm hologram, and vice-versa. A comparison of the pixel count data for the different test configurations shows that the pixel values for the dark region are $92 \%$ lower than the focused image and an out-of-focus image is $72 \%$ lower than the focused imaged. This test verifies that the hologram performs as designed and confirms that each source fills half of the camera aperture.

The resolution performance is measured with both sources turned on. The results are shown in Figs. 4(c) and 4(d) for the surface and depth focus positions, respectively. Upon visual evaluation, both channels are capable of imaging group 8, element $3(322.5 \mathrm{lp} / \mathrm{mm})$, which corresponds to a line pair width of $3.1 \mu \mathrm{m}$. The measured contrast for group 8 elements 1 to 3 is also given. This is an improvement in lateral resolution from the reflectance endoscope system that had a resolution of $4.2 \mu \mathrm{m}$ using curved fringe holograms with the other optics being the same. ${ }^{6}$

Biological samples were used to evaluate imaging depth performance when a tissue volume was illuminated. The resulting images, shown in Fig. 5, utilize postprocessing background subtraction methods in ImageJ software. ${ }^{23}$ Onion skin and mouse fat samples were selected for their large and easily recognizable cellular structures. In addition, low-contrast mouse lung and intestine samples were also imaged and are shown in Fig. 5.

The surface channels for all of the samples show welldefined cellular walls. In the cases of the onion skin and the fat sample, the depth image also provides subcellular details. However, the lung and intestine samples do not provide the 


\section{JBO Letters}

Surface in focus

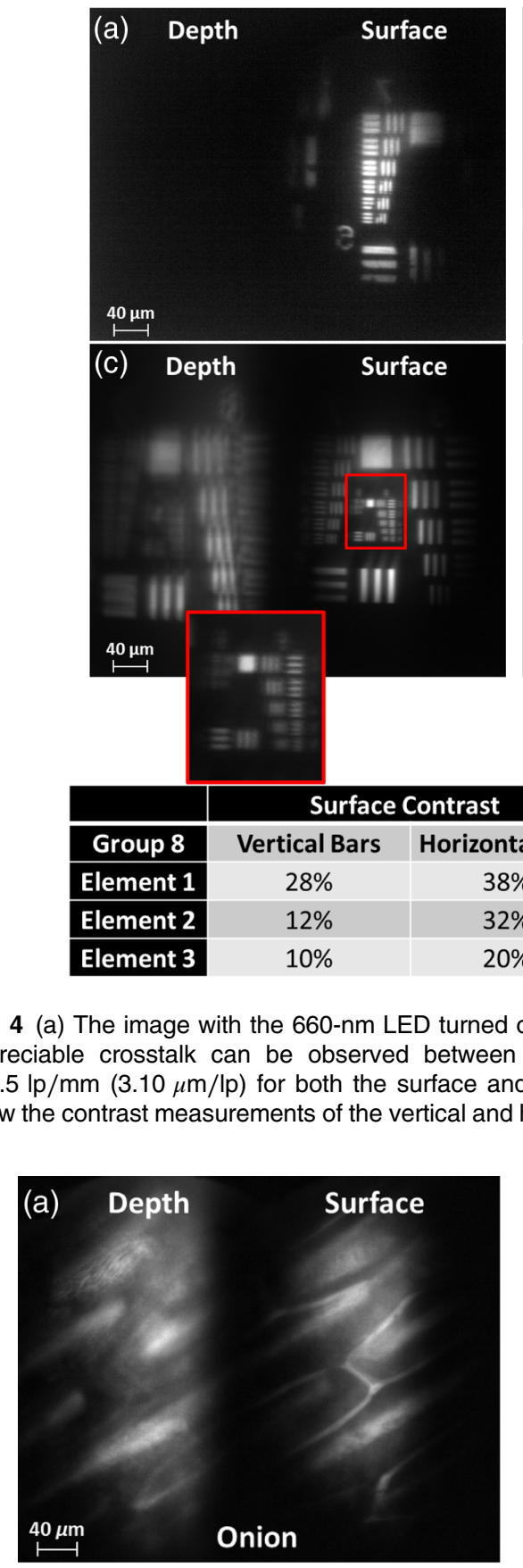
appreciable crosstalk can be observed between the two channels. (c) and (d) A resolution of $322.5 \mathrm{lp} / \mathrm{mm}(3.10 \mu \mathrm{m} / \mathrm{lp})$ for both the surface and the depth channels. The data below the figures show the contrast measurements of the vertical and horizontal bar targets in selected group 8 elements.
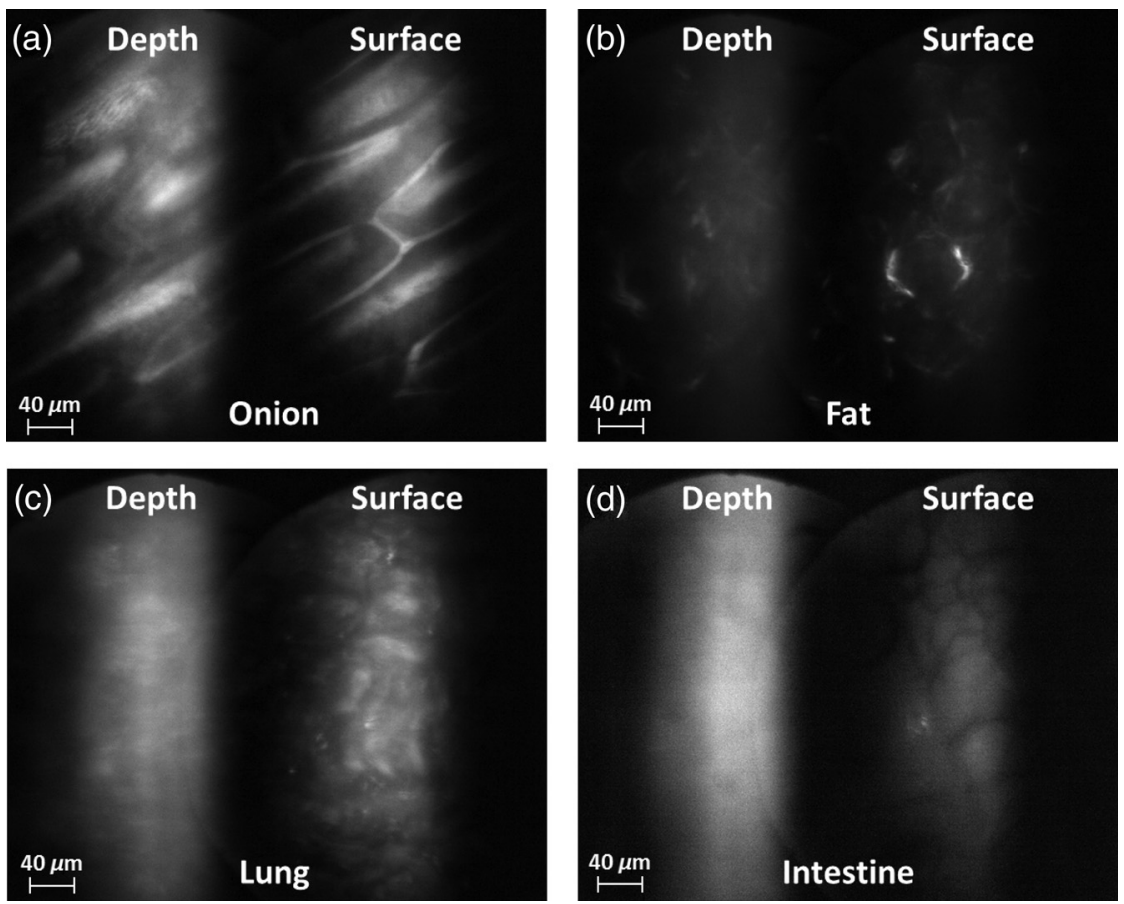

Fig. 5 Biological sample images. (a) Onion skin, (b) mouse fat, (c) mouse lung, and (d) mouse intestine are used in the evaluation. The surface channel in each case shows well-defined cell structure. (a) and (b) Higher contrast samples perform better in the depth channel with more cellular information being present. (c) and (d) Lower contrast and results in loss of feature recognition. 
same contrast or detail for the depth images due to the lower contrast of the tissue.

The presence of the higher background in the depth image can be attributed to lower contrast tissue features and the lack of depth selectivity and background rejection due to Bragg degeneracy of the hologram. The WC-VHI design reduces crosstalk between the channels but does not reject background within a channel. The latter is the result of Bragg degeneracy by the volume hologram because the wavelengths from a source illuminate all positions within the tissue volume. Nonetheless, the WC-VHI system has a lower amount of backscatter than was observed for the reflectance endoscope utilizing only one source.

In summary, initial results indicate that the WC endoscope utilizing an axial chromatic dispersion system is capable of multifocal imaging with greater resolution than the single wavelength reflectance system. ${ }^{6}$ The $100-\mu \mathrm{m}$ difference in image depth is large enough to view variations in the cellular structure of onion skin as well as animal tissue samples such as mouse fat. In addition, the evaluation of lower contrast tissue samples, such as lung and intestine samples, shows significant loss of image feature characteristics at depths of $100 \mu \mathrm{m}$ due to the lack of background rejection within the image channel due to Bragg degeneracy of the hologram and unstructured illumination. In future work, different structured illuminations will be incorporated to eliminate this problem.

\section{Disclosures}

No conflicts of interest, financial or otherwise, are declared by the authors.

\section{Acknowledgments}

This work was supported by a grant from the National Institutes of Health (NIH) (No. R01Ca134424). Isela Howlett received support from the National Science Foundation Bridge to the Doctorate Fellowship, the UA-NASA Space Grant, and the Alfred P. Sloan Foundation (Sloan Foundation, Minority PhD program). The authors would like to acknowledge Caitlin Howard for her assistance during mouse tissue acquisition.

\section{References}

1. A. A. Tanbakuchi et al., "In vivo imaging of ovarian tissue using a novel confocal microlaparoscope," Am. J. Obstet. Gynecol. 202(1), 90.e1-90. e9 (2010).
2. L. P. Hariri et al., "Laparoscopic optical coherence tomography imaging of human ovarian cancer," Gynecol. Oncol. 114(2), 188-194 (2009).

3. J. M. Castro et al., "Confocal-rainbow volume holographic imaging system," Appl. Opt. 50(10), 1382-1388 (2011).

4. E. E. De Leon, "Optical design of volume holographic imaging systems for microscopy," PhD Dissertation, The University of Arizona, Tucson, Arizona (2012).

5. P. J. Gelsinger-Austin et al., "Optical design for a spatial-spectral volume holographic imaging system," Opt. Eng. 49(4), 043001 (2010).

6. I. D. Howlett et al., "Volume holographic imaging endoscopic design and construction techniques," J. Biomed. Opt. 22(5), 056010 (2017).

7. Y. Luo et al., "Simulations and experiments of aperiodic and multiplexed gratings in volume holographic imaging systems," Opt. Express 18(18), 19273-19285 (2010).

8. W. Liu, D. Psaltis, and G. Barbastathis, "Real-time spectral imaging in three spatial dimensions," Opt. Lett. 27(10), 854-856 (2002).

9. W. Liu, G. Barbastathis, and D. Psaltis, "Volume holographic hyperspectral imaging," Appl. Opt. 43(18), 3581-3599 (2004).

10. Y. Luo et al., "Phase-contrast volume holographic imaging system," Opt. Lett. 36(7), 1290-1292 (2011).

11. A. Sinha and G. Barbastathis, "N-ocular volume holographic imaging," Appl. Opt. 43(31), 5784-5795 (2004).

12. J. M. Castro et al., "Confocal rainbow volume holographic imaging," Opt. Photonics News 22(12), 52 (2011).

13. Y. Luo et al., "Multiplexing volume holographic gratings for a spectralspatial imaging system," Proc. SPIE 6912, 69120A (2008).

14. G. Barbastathis and D. J. Brady, "Multidimensional tomographic imaging using volume holography," Proc. IEEE 87(12), 2098-2120 (1999).

15. W. Sun and G. Barbastathis, "Rainbow volume holographic imaging," Opt. Lett. 30(9), 976-978 (2005).

16. Y. Luo, S. B. Oh, and G. Barbastathis, "Wavelength-coded multifocal microscopy," Opt. Lett. 35(5), 781-783 (2010).

17. Y. Luo et al., "Spectrally resolved multidepth fluorescence imaging," J. Biomed. Opt. 16(9) 096015 (2011).

18. P. M. Lane, R. P. Elliott, and C. E. MacAulay, "Confocal microendoscopy with chromatic sectioning," Proc. SPIE 4959, 23-26 (2003).

19. C. Olsovsky et al., "Chromatic confocal microscopy for multi-depth imaging of epithelial tissue," Biomed. Opt. Express 4(5), 732-740 (2013).

20. H. J. Tiziani, "Chromatic confocal microscopy with microlenses," J. Mod. Opt. 43(1), 155-163 (1996).

21. H. Kogelnik, "Coupled wave theory for thick hologram gratings," Bell Labs Tech. J. 48, 2909-2947 (1969).

22. J. M. Castro et al., "Spatial-spectral volume holographic systems: resolution dependence on effective thickness," Appl. Opt. 50(7), 1038-1046 (2011).

23. C. A. Schneider, W. S. Rasband, and K. W. Eliceiri, "NIH Image to ImageJ: 25 years of image analysis," Nat. Methods 9(7), 671-675 (2012). 